\title{
Identification of a Slowly Relaxing Paramagnetic Center in Graphene Oxide
}

\author{
Maria A. Augustyniak-Jabłokow ${ }^{1}$ (D) $\cdot$ Ryhor Fedaruk $^{2} \cdot$ Roman Strzelczyk $^{1}$. \\ Łukasz Majchrzycki ${ }^{3}$
}

Received: 23 June 2018 / Revised: 29 August 2018 / Published online: 14 September 2018

(c) The Author(s) 2018

\begin{abstract}
We demonstrate the existence of two types of paramagnetic centers in a pure graphene oxide. Saturation features of the electron paramagnetic resonance (EPR) spectrum in the temperature range of 4.2-300 K reveal that one of these centers has the spin-lattice relaxation time longer than $1.6 \mu$ s at room temperature. The spectrum of these centers consists of a central line and two satellite lines. The satellite lines result from the forbidden transitions in the hyperfine structure induced by protons in the vicinity of the slowly relaxing centers. The observability of the satellites can be used as a characteristic signature of graphene oxide purity. Since the number of slowly relaxing centers is reduced during chemical reduction of graphene oxide, this type of centers can be attributed to the isolated unfunctionalized carbons in the highly functionalized regions of GO. The second type of paramagnetic centers has shorter relaxation times and dominates in the reduced graphene oxide.
\end{abstract}

\section{Introduction}

Graphene oxide (GO) is a well-known and widely used form of functionalized graphene $[1,2]$. Experimental studies of paramagnetic properties of this material have been reported [3-8]. The electron paramagnetic resonance (EPR) signals of GO and other graphene-related materials can be due to localized electrons, conduction electrons or their exchange coupled system $[9,10]$. Although the saturation of the EPR signal has been observed $[4,8]$, the electron spin relaxation in GO has not been

Maria A. Augustyniak-Jabłokow

maria.augustyniak@ifmpan.poznan.pl

1 Institute of Molecular Physics, Polish Academy of Sciences, Smoluchowski Str. 17, 60-179 Poznan, Poland

2 Institute of Physics, Faculty of Mathematics and Physics, University of Szczecin, Wielkopolska Str. 15, 70-451 Szczecin, Poland

3 Wielkopolska Centre of Advanced Technology, Adam Mickiewicz University, ul. Umultowska 89 C, 61-614 Poznan, Poland 
discussed in detail. Theoretical studies of magnetic properties of some functional groups and their aggregations on the GO surface have shown that nonmagnetic configurations of the functional groups are more energetically favorable [11]. Thermal or chemical removal of functional groups existing on the GO surface produces reduced graphene oxide (RGO), a kind of chemically derived graphene [12, 13]. Such modification extends the range of possible applications of this material [13]. Properties of RGO strongly depend on the reduction method resulting in different parameters of EPR spectra and their temperature dependences [3, 7, 14-16].

Graphene-related 2D materials containing centers with long spin relaxation time are of the great interest for nanotechnologies $[17,18]$. The majority of studies have focused on relaxation of conduction electrons, but their longest relaxation time observed in graphene at room temperature (RT) reaches only 12 ns [19]. Recently, the slow relaxing (1-3 $\mu \mathrm{s})$ spin components in the EPR spectra of chemically exfoliated graphites and a reduced graphene oxide have been identified by pulsed EPR at $80 \mathrm{~K}$, but density of these centers was very low [20].

In this paper, we report our continuous wave EPR study of a very pure GO and RGO. We used the rapid passage effects under microwave saturation to identify paramagnetic centers with the spin-lattice relaxation time $\left(T_{1}\right)$ longer than $1 \mu \mathrm{s}$ at RT in GO. Another type of centers with short relaxation times is also observed in this material. These fast relaxing centers dominate in RGO.

\section{Experimental}

GO was obtained from a large-flake natural graphite using the modified Hummer method [21], as it was described elsewhere [5]. The product was purified to decrease the contamination level to $6 \mathrm{ppm}$ for $\mathrm{Mn}^{2+}$ and $3 \mathrm{ppm}$ for $\mathrm{Fe}^{3+}$. The studied samples were in a form of GO paper, obtained by drying concentrated suspension on the flat surface. RGO was prepared by chemical reduction of GO with the use of ascorbic acid. To slowing down the process and avoid attachment of hydrogen, reduction was carried out in the diluted suspension at low temperature. The spin density in the studied materials was $3 \times 10^{17} \mathrm{spin} / \mathrm{g}$ in GO and $4 \times 10^{16} \mathrm{spin} / \mathrm{g}$ in RGO as was determined with using the standard sample.

EPR measurements were performed using a continuous wave (CW) X-band RADIOPAN SX spectrometer with Oxford ESR900 cryostat at $4.2 \mathrm{~K}$ and at RT. The amplitude of the $100 \mathrm{kHz}$ magnetic field modulation was $0.01 \mathrm{mT}$. The absorption CW EPR spectrum is usually obtained by phase-sensitive detection at the frequency, $\omega_{\text {rf }}$, of the magnetic field modulation, which selects the first-harmonic component of the spin response, even though higher harmonics are present [22-24]. In-phase and $90^{\circ}$ out-of-phase (or simply out-of-phase) components refer to the phase of the EPR signal relative to that of the magnetic field modulation. Typically, CW EPR spectra are recorded in the absence of microwave (MW) saturation under slow passage conditions, where the rate of change of the magnetic field due to sweep of the external magnetic field, $B$, and to magnetic field modulation, $B_{\mathrm{m}}$, is slow relative to the spin relaxation rates [22]. When the first-harmonic in-phase signal is detected, the first 
derivative of the EPR absorption signal is recorded as a function of $B$. Under these conditions, the first-harmonic out-of-phase signal is averaged to zero [23].

"Rapid passage" through resonance occurs when the rate of change of $B$ or $B_{\mathrm{m}}$ is greater than the electron spin relaxation rate [25]. In this case, the spin response lags the modulating field and the first-harmonic out-of-phase signal is generated under microwave power saturation. These out-of-phase signals are rapid passage signals and their observation reveals that the spin lattice relaxation time, $T_{1}$, of the spin system is of the order of $1 / \omega_{\mathrm{rf}}(1.6 \mu \mathrm{s}$ at $100 \mathrm{kHz})$ or longer. At high microwave power, the out-of-phase signal can be more intensive than the in-phase one [25].

We used the appearance of the out-of-phase EPR signal to reveal paramagnetic centers with long $(>1.6 \mu \mathrm{s})$ relaxation times. For accurate phase settings of phasesensitive detector, a non-saturable reference sample was used. The reference sample was a small single crystal of $\mathrm{CuSO}_{4} \cdot 5 \mathrm{H}_{2} \mathrm{O}$, which was attached at the side of the $\mathrm{GO}$ sample. There is no out-of-phase signal from the fast relaxing $\mathrm{Cu}^{2+}$ ions (Fig. 1a). The out-of-phase signal was observed only for the GO sample (Fig. 1a, b). The MW saturation behaviors of the in-phase and out-of-phase EPR signals were studied to identify the origin of the observed paramagnetic centers.

\section{Results and Discussion}

Figure 2 shows saturation of the EPR signal for the GO and RGO samples. The in-phase and out-of-phase signals of both materials, recorded at RT and MW power $P$ of $5.8 \mathrm{~mW}$, are presented in Fig. 2a. The saturation results in the nonlinear dependence of the EPR signal intensity on the square root of MW power as it is observed for the in-phase and out-of-phase signals of GO (Fig. 2b). The out-of-phase signals saturate at higher MW power than the in-phase ones. The increase of the in-phase EPR signal intensity of GO at RT and $P \geq 21 \mathrm{~mW}$ indicates the contribution of additional paramagnetic centers with shorter $T_{1}$ (Fig. 2b). Thus, the observed saturation curve demonstrates the existence of two types of paramagnetic centers with different spin relaxation times in the GO sample. At RT, the in-phase signal of RGO shows nearly linear $I\left(P^{1 / 2}\right)$ dependence. This
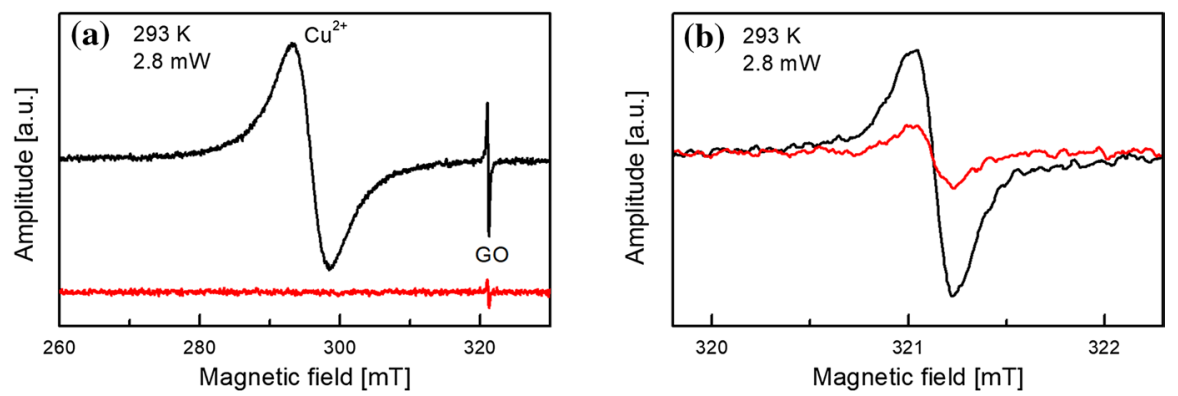

Fig. 1 a The appearance of the out-of-phase EPR signal in the GO sample. The spectra were recorded for the sample composed of a small $\mathrm{CuSO}_{4} \cdot 5 \mathrm{H}_{2} \mathrm{O}$ crystal and $\mathrm{GO}$ paper. The black and red lines show the in-phase and out-of-phase signal, respectively. b A zoom of the narrow GO signal presented in a (color figure online) 

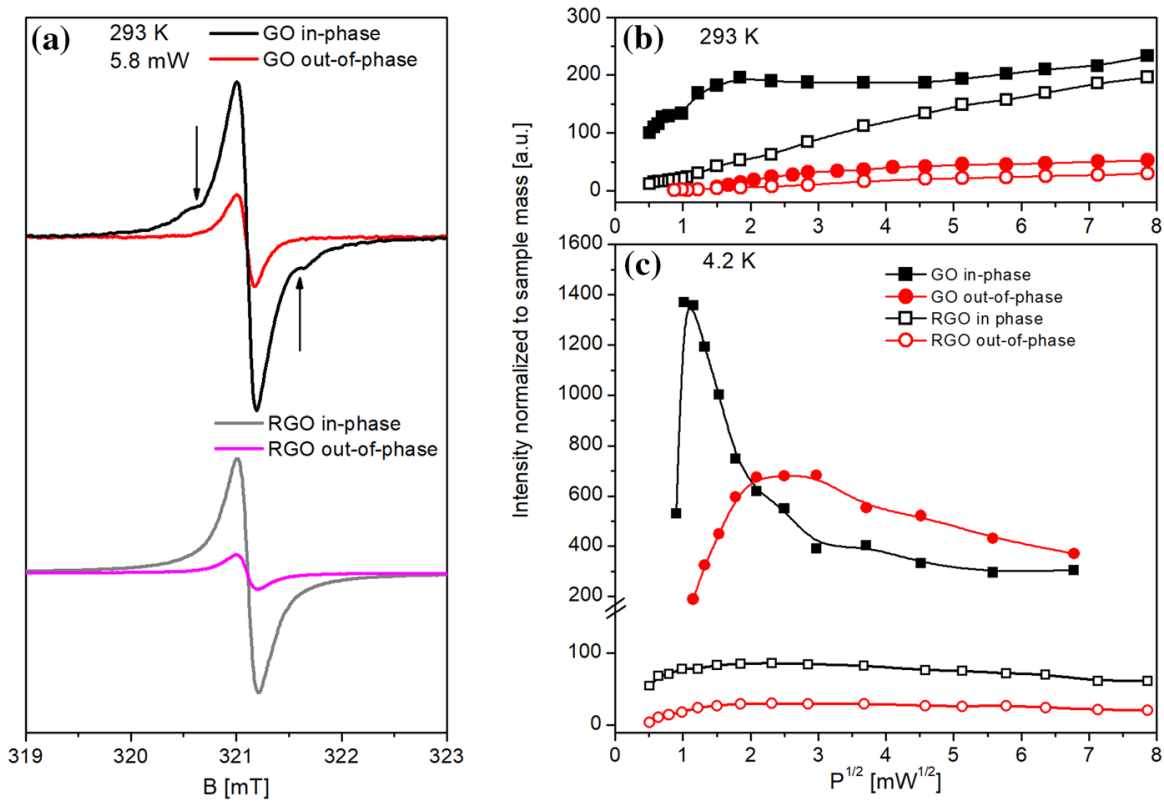

Fig. 2 a In-phase and out-of-phase signals of GO and RGO samples recorded at MW power of $5.8 \mathrm{~mW}$ at RT. Positions of the satellite lines are shown by arrows. b and $\mathbf{c}$ Saturation of the EPR signals for the GO and RGO samples at RT and $4.2 \mathrm{~K}$, respectively. The signal intensities were calculated from $I=A \Delta B_{\mathrm{pp}}^{2}$, where $A$ is a peak-to peak amplitude and $\Delta B_{\mathrm{pp}}$ is the signal peak-to-peak linewidth

indicates that the EPR spectrum of RGO is dominated by the centers with the short spin relaxation time. Although the concentration of spins in RGO is almost ten times lower than that in GO, the weak saturation causes that at high MW powers the intensity of the GO and RGO signals are comparable (Fig. 2b). Comparison of the saturation curves of GO and RGO suggests that the same type of the fast-relaxing centers exists in both materials. Differences in the spin concentration and in the character of the $I\left(P^{1 / 2}\right)$ dependencies suggest that the number of the slowly relaxing centers, which dominates EPR spectra of GO, is significantly decreased by the reduction process. Temperature lowering to $4.2 \mathrm{~K}$ increases the saturation of the EPR signal of both samples (Fig. 2c), indicating that the relaxation times of the all observed centers become longer. The out-of-phase signal in RGO weakly depends on MW power and temperature. We cannot exclude that this signal is due to the residual amount of the slowly relaxing and strongly saturating centers undetected in the in-phase EPR signal.

A characteristic feature of the observed EPR spectra of GO is the existence of a partially resolved pair of weak satellites on either side of the central line (Fig. 2a). The satellite lines are separated by $0.96 \pm 0.01 \mathrm{mT}$. The observed relative intensities of the satellites and the central line depend on MW power and temperature and are different for the in-phase and out-of-phase signals. The satellite lines saturate at higher MW power than the central line. Therefore, they become more visible when 
the central line is significantly saturated. According to our experience, these satellite lines can result from the forbidden transitions in the hyperfine structure due to protons in the vicinity of the paramagnetic center [26, 27].

The saturation properties of the observed central EPR line and its satellites as well as the value of their splitting confirm that the EPR spectrum results from a paramagnetic defect with a well-defined hyperfine interaction arising from a single hydrogen atom $[22,28]$. The unpaired electron produces the hyperfine magnetic field $\boldsymbol{B}_{\mathrm{hf}}=\boldsymbol{A} \boldsymbol{B} / 2 g_{\mathrm{N}} \mu_{\mathrm{N}} B$ at the hydrogen nucleus, where $g_{N}$ is the proton $\mathrm{g}$-factor, $\mu_{\mathrm{N}}$ is the nuclear magneton, and $\boldsymbol{A}$ is the nuclear hyperfine tensor. When the distance between the electron and the proton is $0.15-0.5 \mathrm{~nm}$, the hyperfine field $B_{\mathrm{hf}}$ is comparable with the external magnetic field $\boldsymbol{B}$. Vector addition of the external and hyperfine fields gives the effective magnetic field $\boldsymbol{B}_{\text {eff }}$. When the nuclear hyperfine tensor $\boldsymbol{A}$ is anisotropic, two vector components of $\boldsymbol{B}_{\text {eff }}$ can point in different directions. As it is shown in Fig. 3a, $\theta$ is the angle between the nuclear quantization axis for the electron $M_{S}=+1 / 2$ state $\left(\boldsymbol{B}-\boldsymbol{A} \boldsymbol{B} / 2 g_{\mathrm{N}} \mu_{\mathrm{N}} B\right)$ and that for the $M_{\mathrm{S}}=-1 / 2$ state $\left(\boldsymbol{B}+\boldsymbol{A} \boldsymbol{B} / 2 g_{\mathrm{N}} \mu_{\mathrm{N}} B\right)$. At constant external magnetic field $B$, which is comparable with $B_{\mathrm{hf}}$, the hyperfine interactions lead to four energy levels associated with the electron $M_{\mathrm{S}}= \pm 1 / 2$ and proton $M_{\mathrm{N}}= \pm 1 / 2$ states (Fig. 3b). It is known $[22,28]$ that the intensity of the allowed $\left(\Delta M_{\mathrm{N}}=0\right)$ transitions is proportional to $\cos ^{2}(\theta / 2)$ and the intensity of the forbidden $\left(\Delta M_{\mathrm{N}}= \pm 1\right)$ transitions is proportional to $\sin ^{2}(\theta / 2)$. The angle $\theta$ between $\boldsymbol{B}_{\text {eff }}(+)$ and $\boldsymbol{B}_{\text {eff }}(-)$ depends on the anisotropy in $\boldsymbol{A}$, which is proportional to $2 g_{\mathrm{N}} \mu_{\mathrm{N}} B$. A large value of $\theta$ leads to significant intensity

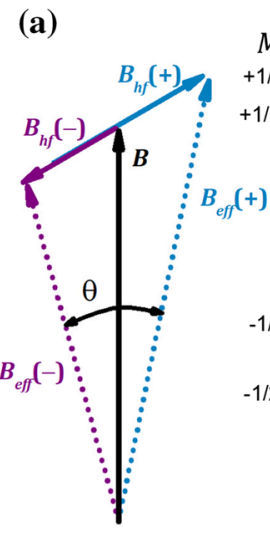

(b)

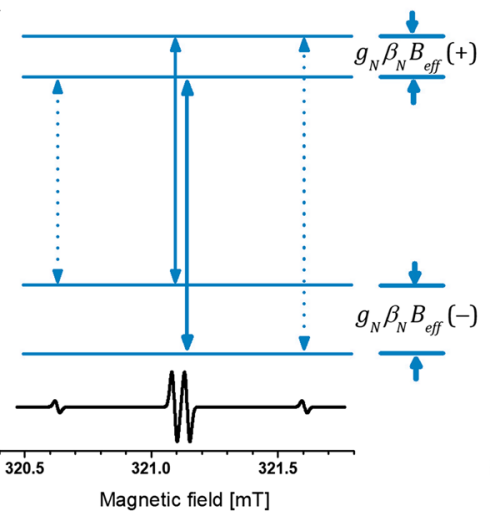

(c)

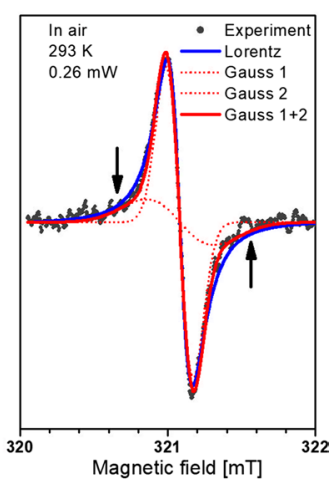

Fig. 3 Satellite lines in the EPR spectra of GO are the forbidden transitions between energy levels resulting from the hyperfine interaction between one unpaired electron and one proton. a The unpaired electron produces the hyperfine magnetic field $B_{\mathrm{hf}}$ at the proton. b Energy levels at constant external magnetic field $B$ which is comparable to $B_{\mathrm{hf}}$. When the nuclear Zeeman term $g_{\mathrm{N}} \mu_{\mathrm{N}} B$ is larger than the hyperfine splitting of the allowed transitions, the forbidden $\Delta M_{\mathrm{I}}= \pm 1$ transitions (dashed lines) are observed as the satellite lines. Expected EPR spectrum is shown in the bottom part. $\mathbf{c}$ The in-phase EPR signal observed for the GO sample at $P=0.26 \mathrm{~mW}$. The hyperfine splitting of the allowed transitions is not resolved. At this MW power, the satellite lines are practically invisible. Due to inhomogeneous broadening the observed signal is better fitted with two Gaussian lines (red lines) than with the Lorentzian lineshape (blue line) (color figure online) 
in the forbidden $\Delta M_{I}= \pm 1$ transitions (dashed lines). These transitions are usually of much lower intensity than the allowed transitions (solid lines). When the nuclear Zeeman term $g_{\mathrm{N}} \mu_{\mathrm{N}} B$ is much larger than the hyperfine splitting of the allowed transitions, their EPR lines are not resolved and they are observed as the single central line. The satellites arise from forbidden $\Delta M_{\mathrm{N}}= \pm 1$ proton spin flips in the hydrogen atom during the EPR $\Delta M_{\mathrm{S}}= \pm 1$ transitions. For protons the satellite positions are at the magnetic field values of $\pm\left(g_{\mathrm{N}} \mu_{\mathrm{N}} / g \mu_{\mathrm{B}}\right) B_{0}$ to either side of the central line. Here $g$ is the electron $g$-factor, $\mu_{\mathrm{B}}$ is the Bohr magneton, and $B_{0}$ is the resonant field for the central line. For $B_{0}=321 \mathrm{mT}$, we find that $\pm\left(g_{\mathrm{N}} \mu_{\mathrm{N}} / g \mu_{B}\right) B_{0}= \pm 0.488 \mathrm{mT}$. These calculated values agree well with the observed satellite positions separated by $0.96 \mathrm{mT}$ (Fig. 2a). The appropriate theory of the allowed and forbidden EPR transitions can be found in $[22,28]$. The hydrogen-related defects with the EPR properties similar to those observed in our GO sample have been studied in the polycrystalline diamonds [28]. The above-discussed theory of the hyperfine structure describes interactions of the unpaired electron with one nucleus. When the larger number of interacting nuclei exists, the hyperfine splitting multiplies and can cause the inhomogeneous dipolar broadening of the EPR signals. Due to this broadening, the hyperfine splitting of the central line is not resolved (Fig. 2a).

Removal of the slowly relaxing centers in the process of the chemical reduction of GO indicates that these centers are located in the highly functionalized regions of GO. We attribute the slowly relaxing centers to the isolated unfunctionalized carbons surrounded by the functionalized ones with the different hybridization. Such localization explains the slow dissipation of energy from these centers to the lattice and their long spin-lattice relaxation time.

To identify the structure of the centers with shorter relaxation times observed in the GO and RGO samples, further investigations are needed.

The dipole-dipole hyperfine interactions, described above, influence properties of the EPR signal. Due to the unresolved hyperfine structure, the observed EPR line is inhomogeneously broadened. As it is shown in Fig. 3c, the observed apparent Lorentzian lineshape can result from overlapping of two Gaussian signals. This strongly impedes qualitative analysis of the saturation profiles under rapid passage condition $\left(\omega_{r f} T_{1} \gg 1\right)$. It is known that the values of the spin-lattice $\left(T_{1}\right)$ and spin-spin $\left(T_{2}\right)$ relaxation times can be obtained by the simulation of the saturation profiles. At the slow passage and the known MW field amplitude $B_{1}$, there is the analytical solution of the Bloch equations for such determination [see for example 25, 29]. Under rapid passage, the modification of the Bloch equations by taking into account the amplitude of MW field $B_{1}$, the amplitude $B_{\mathrm{m}}$ and frequency of modulation enables determination of the relaxation times from saturation curves [25, 30-32]. In our case only numerical solutions of the modified Bloch equations can be obtained. However, problems with the estimation of $T_{2}$ from the inhomogeneous linewidth for our samples and with the determination of $B_{1}$ result in the unnecessarily complicated procedures for finding values of the relaxation times. Due to specific properties of GO and RGO, the value of $B_{1}$ depends not only on the MW power and the properties of the resonator, but also on the MW-dependent properties of the samples. The functional groups on the GO surface are gathered in clusters. The highly functionalized nonconducting regions of $\mathrm{sp}^{3}$ hybridization coexist with graphene-like electrically 
conducting regions of $\mathrm{sp}^{2}$ dominant hybridization. The RGO sample is a form of slightly functionalized, therefore, defected graphene. Even when the samples are carefully placed in the maximum magnetic MW field, the increase in MW power inevitably leads to the influence of the electric MW field on our bulk samples. In graphene, the electric field generates current carriers, free electrons and holes, and their number is proportional to the electric field. Increasing the number of the paramagnetic centers as well as growing losses due to the cyclotron motion of conduction electrons in the external magnetic field $B_{0}$ change $B_{1}$. Moreover, due to hygroscopic properties of GO water molecules can be adsorbed on its surface [33] and the proton conductivity of this material can be observed [6, 34, 35]. This conductivity as well as the molecular dynamics of the functional groups on the surfaces of GO and RGO result in the non-resonant losses and can also change $B_{1}$. Therefore, in this paper, we find only the limit for $T_{1}$ and the values of the relaxation times will be determined in future pulsed EPR studies.

\section{Conclusions}

In conclusion, we have demonstrated the existence of two types of paramagnetic centers in GO. One of these centers has the spin-lattice relaxation time longer than $1.6 \mu$ s at room temperature. The second type of paramagnetic centers with shorter relaxation times is revealed at high MW power. The observed lineshape of the EPR signal is due to overlapping signals of two types of centers and is inhomogeneously broadened. The observed satellite lines in the EPR spectra show the presence of protons in the vicinity of the slowly relaxing centers. The satellites can be used as a signature of GO purity, because their detection is impeded by contamination with paramagnetic ions $\left(\mathrm{Mn}^{2+}, \mathrm{Fe}^{3+}\right.$ and others), which decreases the relaxation times and results in the broadening of the EPR signal. The chemical reduction removes the slowly relaxing centers and the fast relaxing centers dominate in RGO.

Acknowledgements This work was partially funded by the Polish National Science Centre, Grant no. 2016/21/D/ST3/00975.

Open Access This article is distributed under the terms of the Creative Commons Attribution 4.0 International License (http://creativecommons.org/licenses/by/4.0/), which permits unrestricted use, distribution, and reproduction in any medium, provided you give appropriate credit to the original author(s) and the source, provide a link to the Creative Commons license, and indicate if changes were made.

\section{References}

1. D.R. Dreyer, S. Park, C.W. Bielawski, R.S. Ruoff, Chem. Soc. Rev. 39, 228 (2010)

2. A.M. Dimiev, S. Eigler (eds.), Graphene Oxide: Fundamentals and Applications (Wiley, Hoboken, 2016)

3. L. Ćirić, A. Sienkiewicz, D.M. Djokić, R. Smajda, A. Magrez, T. Kaspar, R. Nesper, L. Forró, Phys. Status Solidi B 247, 2958 (2010)

4. A.M. Panich, A.I. Shames, N.A. Sergeev, Appl. Magn. Reson. 44, 107 (2013) 
5. Ł. Majchrzycki, M.A. Augustyniak-Jabłokow, R. Strzelczyk, M. Maćkowiak, Acta Phys. Pol. A 127, $540(2015)$

6. M. Kempiński, S. Łoś, P. Florczak, W. Kempiński, S. Jurga, Acta Phys. Pol. A 132, 81 (2017)

7. A. Diamantopoulou, S. Glenis, G. Zolnierkiwicz, N. Guskos, V. Likodimos, J. Appl. Phys. 121, 043906 (2017)

8. P. Stathi, D. Gournis, Y. Deligiannakis, P. Rudolf, Langmuir 31, 10508 (2015)

9. M.A. Augustyniak-Jabłokow, K. Tadyszk, M. Maćkowiak, S. Lijewski, Chem. Phys. Lett. 557, 118 (2013)

10. M.A. Augustyniak-Jabłokow, K. Tadyszk, M. Maćkowiak, Y. Yablokov, Phys. Status Solidi RRL 5, 271 (2011)

11. N. Ghaderi, M. Peressi, J. Phys. Chem. C 114, 21625 (2010)

12. C.K. Chua, M. Pumera, Chem. Soc. Rev. 43, 291 (2014)

13. S. Pei, H.M. Cheng, Carbon 50, 3210 (2012)

14. L. Ćirić, A. Sienkiewicz, R. Gaál, J. Jaćimović, C. Vâju, A. Magrez, L. Forro, Phys. Rev. B 86, 195139 (2012)

15. A.M. Panich, A.I. Shames, A.E. Aleksenskii, A. Dideikin, Solid State Commun. 152, 466 (2012)

16. M. Kempiński, P. Florczak, S. Jurga, M. Śliwińska-Bartkowiak, W. Kempiński, Appl. Phys. Lett. 111, 084102 (2017)

17. D. Pesin, A.H. MacDonald, Nat. Mater. 11, 409 (2012)

18. C. Kloeffel, D. Loss, Annu. Rev. Condens. Matter Phys. 4, 51 (2013)

19. M. Drögeler, C. Franzen, F. Volmer, T. Pohlmann, L. Banszerus, M. Wolter, K. Watanabe, T. Taniguchi, C. Stampfer, B. Beschoten, Article. Nano Lett 16, 3533 (2016)

20. A. Barbon, F. Tampieri, AIMS Mater. Sci. 4, 147 (2017)

21. W. Hummers, R.J. Offeman, Am. Chem. Soc. 80, 1339 (1958)

22. J.A. Weil, J.R. Bolton, Electron Paramagnetic Resonance: Elementary Theory and Practical Applications, 2nd edn. (Wiley, Hoboken, 2007)

23. M. Kälin, I. Gromov, A. Schweiger, J. Magn. Reson. 160, 166 (2003)

24. A.P. Saiko, R. Fedaruk, S.A. Markevich, J. Magn. Reson. 259, 47 (2015)

25. J.R. Harbridge, G.A. Rinard, R.W. Quine, S.S. Eaton, G.R. Eaton, J. Magn. Reson. 156, 41 (2002)

26. M.A. Augustyniak, S.K. Hoffmann, J. Goslar, W. Hilczer, J. Wolak Ferroelectr. Lett. 15, 69 (1993)

27. W. Hilczer, S.K. Hoffmann, J. Goslar, J. Tritt-Goc, M.A. Augustyniak, Solid State Commun. 85, 585 (1993)

28. X. Zhou, G.D. Watkins, K.M. Rutledge, R.P. Messmer, S. Chawla. Phys. Rev. B 54, 7881 (1996)

29. T.G. Castner, Phys. Rev. 115, 1506 (1959)

30. M. Weger, Bell Syst. Tech. J. 39, 1013 (1960)

31. V.A. Livshits, T. Pa'li, D. Marsh, J. Magn. Reson. 134, 113 (1998)

32. M. Marrale, A. Longo, M. Brai, A. Barbon, M. Brustolon, Radiat. Res. 181, 184 (2014)

33. R. Strzelczyk, C.E. Giusca, F. Perrozzi, G. Fioravanti, L. Ottaviano, O. Kazakova, Carbon 122, 168 (2017)

34. M.R. Karim, K. Hatakeyama, T. Matsui, H. Takehira, T. Taniguchi, M. Koinuma, Y. Matsumoto, T. Akutagawa, T. Nakamura, S. Noro, T. Yamada, H. Kitagawa, T. Yamada, J. Am. Chem. Soc. 135, 8097 (2013)

35. K. Hatakeyama, H. Tateishi, T. Taniguchi, M. Koinuma, T. Kida, S. Hayami, H. Yoko, Y. Matsumoto, Chem. Mater. 26, 5598 (2014) 